\title{
Structure-Guided Biochemical Analysis of Quorum Signal Synthase Specificities
}

Shi-Hui Dong, Mila Nhu-Lam, Rajesh Nagarajan, Satish K. Nair

\section{Supplementary Information}

Page

\section{Material and Methods}

Table S1: Data collection, phasing and refinement statistics

Table S2: The information of LuxI AHL synthases

Table S3: Primers used for generating variants

Figure S1: Closeup view of the overlay of CoA binding site of RpaI and BjaI

Figure S2: Sequence alignment analysis of cluster 1b

Figure S3: The kinetics curves and parameters of RpaI, BolI, and MaqI

Figure S4: The bar diagrams of catalytic efficiency of MesI and MplI

Figure S5: The kinetics curves of MesI, MplI, BolI and RpaI

Figure S6: Sequence alignment analysis of cluster 1c

Figure S7: High Resolution LC-MS analysis of MaqI reaction.

Figure S8: The kinetics curves of MplI

Figure S9: Sequence alignment analysis of cluster 2

Figure S10: ESI mass spectra of RpaI variant reactions

Figure S11: ESI mass spectra of MesI variant reactions

Figure S12: ESI mass spectra of BjaI variant reactions

Figure S13: ESI mass spectra of MplI variant reactions

References 


\section{Materials and Methods}

\section{General methods}

Oligonucleotides were purchased from Integrated DNA Technologies. Restriction endonucleases, DNA polymerases, T4 DNA polymerase were obtained from New England Biolab. Amicon Ultra-4 centrifugal filters were ordered from MILLIPORE. Chemicals were ordered from Sigma Aldrich. E. coli DH5 $\alpha$ and E. coli Rosetta 2 cells were used as host for cloning and plasmid propagation, and host for protein expression, respectively. Expression vectors were obtained from Addgene.

The gene encoding MaqI was synthesized as gBlocks Gene Fragments (Integrated DNA Technologies) with codon optimization for E. coli expression. All polymerase chain reactions (PCR) were carried out on a Mastercycler thermal cycler (Eppendorf). Amplification primers (Table S1) were designed based on the published sequences. DNA sequencing was performed by ACGT, Inc.

MaqI gene sequence:

ATGATCCGGCTTCACGCTATAGATAGAACATCGAGAGGCGCCTACGCTGACGCTCT TGCCCAGCACCATGCTTTGAGACGTGCAGCCTTTGAAGAAGGTAGATGTCGTGCCCTTC GGAGCGTAGATGCCGCCGGTCGTGATCGCTTCGATACGGAGGCCACCCGTTATCTGTTG GCTATAGACGGGGACGGCCGTGTCGCTGGAGGGACTCGGCTGCTGCCTAGTGATGGCC CGACTCTTCTTAACGCGGTTTTTCCACATCTGGCTGACATTCGGGGGTTGCCTCGTGAA CCCGGTACTTGGGAATGTACGCGGTTGTTCTCCAGTACCCGTTATCGTGAAGAACGTCC GTTGAGCCGGGCCGCTGGGATTGTCTCGGCCGGTATGATCGAGCATTGCTTGGAACAG GGCATCCCTTACTTGTCATTAGTCGTCGAAACATACTGGATTCCCCAGTTGGCGGAAAT AGGATGGCGCCCACGCCCTCTTGGTTTACCCGTTGCCTGCGAGGGGGTCTCATTATGCG CAGTGAGCATCGCCATGTCCGAAGAAGCGCTGCGGGAAACTCGGGCGGCATATGGAAT TGTCGCGTCAGTGTTGGCTCGTACGCCCCCTCCGCCGGATAGAACCCGCGCCGGACGTG GTCTTGCTGCG

\section{Crystallization and data collection of RpaI and MesI and homology modelling of MplI}

All proteins are purified using a published protocol. ${ }^{1}$ Size-exclusion chromatographically purified sample of RpaI was concentrated to $3-5 \mathrm{mg} / \mathrm{mL}$ and used for crystallization by the hanging drop vapor diffusion. RpaI was incubated with different ligands $(2 \mathrm{mM})$ for $30 \mathrm{~min}$ before mixing with precipitant solutions in a 1:1 ratio (v/v). The precipitant solution consists of $0.1 \mathrm{M}$ BIS-TRIS PH 5.5 and $25 \%$ w/v polyethylene glycol 3350. RpaI crystals were equilibrated with the precipitant solution supplemented with additional $10 \%$ of w/v polyethylene glycol 3350 , prior to vitrification by direct immersion in liquid nitrogen. MesI $(8 \mathrm{mg} / \mathrm{mL})$ were crystallized in two conditions. The first solution contains $0.1 \mathrm{M}$ sodium cacodylate PH 6.5, 40 \% v/v MPD, and 5 \% w/v PEG 8000. 
The second solution contains $0.1 \mathrm{M}$ sodium cacodylate PH 6.5 and 35\% (v/v) 2-Ethoxyethanol. MesI crystals were flash frozen in liquid nitrogen without additional cryo protection. All data were collected at LS-CAT (Sector 21, Advanced Photon Source, Argonne National Labs, IL) using MAR CCD or MX-300 detectors. All data were integrated and scaled using either HKL2000 ${ }^{2}$ or XDS 3 .

Crystallographic phases were determined by the molecular replacement method as implemented in Phaser $^{4}$ using the coordinates of Bjal (PDB: 5W8D, 5W8G, 5W8C, 5W8A, and $5 \mathrm{~W} 8 \mathrm{E})$. For each structure, iterative model building was carried out using phenix-refine ${ }^{5}$ and further improved by manual fitting and adjustment using COOT. ${ }^{6}$ Cross-validation, using $5 \%$ of the data for the calculation of the free R factor, ${ }^{7}$ was utilized throughout model building process in order to monitor building bias. The stereochemistry of all of the models was routinely monitored throughout the course of refinement using PROCHECK. ${ }^{8}$ Relevant data collection and refinement parameters are provided in Table S2. The coordinates for the RpaI and MesI structures can be accessed under code $6 \mathrm{WN} 0$ and $6 \mathrm{WNS}$.

The homology model of MplI was generated using online tool SWISS-MODEL (https://swissmodel.expasy.org/) with RpaI structure (PDB: 6WN0) as the molecular template. ${ }^{9}$ The output coordinate was used to produce the corresponding figure without any further manipulation. The sequence identity and similarity between MpII and RpaI are 38.6\% and 40.0\%, respectively, over $85 \%$ coverage. The QMEAN and All Atom Z-scores are -2.02 and -1.23, respectively, close to zero, indicating good agreement between the MplI model structure and RpaI experimental template. ${ }^{10}$

\section{Kinetics of RpaI, MesI, BolI, MplI, and MaqI}

DCPIP assay for RpaI, MesI, and BolI. The enzymatic reactions catalyzed by RpaI, MesI, and BolI were monitored using a colorimetric assay (DCPIP microplate) ${ }^{11}$ that is sensitive to the free thiol generated upon transfer of the acyl group from various acyl-CoAs. A typical reaction (100 $\mu \mathrm{L}$ ) contained $30 \mu \mathrm{M}$ DCPIP, $100 \mathrm{mM}$ HEPES pH 7.2, fixed SAM concentration at $500 \mu \mathrm{M}$, and varied acyl-CoA concentrations $(2-150 \mu \mathrm{M})$. To eliminate background rates, SAM, acyl-CoA, HEPES buffer, and DCPIP were incubated for 10 minutes before initiating the reaction with the addition of enzymes. Reactions were initiated by the addition of appropriate enzyme concentration. The thiol-dependent reduction of DCPIP was monitored at $600 \mathrm{~nm}\left(\Delta \varepsilon_{600}=21,000 \mathrm{M}^{-1} \mathrm{~cm}^{-1}\right)$ for 600 seconds. The initial rate data was fit to Michaelis-Menten or substrate inhibition equation using GraphPad Prism 7.0. All experiments were done in triplicate to check for reproducibility and to estimate errors.

HPLC Lactonization assay for Mp1I and MaqI. Lactonization ${ }^{12}$ reaction products could be resolved independently using analytical C-18 reverse-phase UHPLC. The lactonization reaction was monitored using a gradient beginning at $100 \% \mathrm{~A}(99.9 \%$ water, $0.1 \%$ formic acid) and ending at 
$70 \%$ A: $30 \%$ B (99.9\% acetonitrile, $0.1 \%$ formic acid) over a period of 10 minutes at flow rate of $500 \mu \mathrm{L} / \mathrm{min}$. Standard calibration curve for MTA was generated by serial dilution of MTA concentrations in buffer with quench solution, $6 \mathrm{M} \mathrm{HCl}$. Peak areas at each MTA concentration were determined from HPLC chromatograms at $260 \mathrm{~nm}$ (SAM and MTA). A typical enzymecatalyzed reaction $(100 \mu \mathrm{L})$ contained $100 \mathrm{mM}$ HEPES pH 7.3 buffer, fixed SAM concentration at $500 \mu \mathrm{M}$, varied acyl-CoA concentrations $(2-150 \mu \mathrm{M})$. The mixture was split into two equal portions for background and reaction. After incubation at room temperature for 5 minutes, the reaction and background aliquots were initiated, respectively with the appropriate enzyme concentration and water. Both aliquots were quenched after 4 minutes of initiation using $6 \mathrm{M} \mathrm{HCl}$ with final concentration of $0.4 \mathrm{M} \mathrm{HCl}$. Peak areas of MTA at each acyl-CoA concentration were quantified by using Chromeleon 7.2 software. The difference between background and reaction in peak area per minute was converted to initial reaction rate in $\mathrm{M} / \mathrm{s}$ using standard calibration curves. MTA standard curve had linear fit equation of $y=0.198 x$, where $y=$ peak area of MTA, $x=$ known concentration of MTA. The initial rate was fitted to Michaelis-Menten equation or substrate inhibition equation using GraphPad Prism 7.0. All experiments were repeated in duplicate to check for reproducibility and to estimate errors.

\section{Structure Based Engineering of RpaI, MesI, MpII, and BjaI, as well as Their Catalytic Efficacy Evaluation}

Based on the structural information, we created RpaI-Q124A, MesI-L153A, MesI-L153F, MplI-T105Y, and BjaI-F147Y variants, which were purified using the same protocol. ${ }^{1}$ Their activities were evaluated together with the wild type proteins using different acyl-CoAs as substrates. The assay condition was same with previous study, ${ }^{1}$ except for using a mixture of acylCoAs with 1:1 ratio to evaluate the substrate specificity. The substrate specificity of RpaI and its Q124A variant were evaluated using mixture of $p \mathrm{C}-\mathrm{CoA}$ and 4-OMe-cinnamoyl-CoA (Me- $p \mathrm{C}$ CoA). Wild type MesI was tested using mixture of C4-, C6-, and C8-CoAs, while the mixture of C6- and C8-CoAs was used for its L153A variant, and the mixture of C4- and C6-CoAs was used for its $\mathrm{L} 153 \mathrm{~F}$ variant. The substrate specificity of MplI and its T105Y variant was evaluated using the mixture of C12- and C4-CoAs. The substrate specificity of BjaI and its F147Y variant was studied using the mixture of IV- and C4-CoAs.

After overnight incubation, all reactions were quenched by adding same volume of acetonitrile with $0.1 \%$ of formic acid, and then spun down to remove the precipitate. Each of the reaction supernatant was subject to mass analysis (positive mode) using Agilent 1200 series HPLC equipped with an Agilent G1956B mass spectrometer. The production of each AHLs in each reactions was recorded based on their $\mathrm{m} / \mathrm{z}[\mathrm{M}+1]^{+}$peak intensities from SIM mode, and the total amount of AHL 
mixture was normalized to $1(100 \%)$ and each of the AHL percentage reflected its substrate preference. 
Table S1. Data collection, phasing and refinement statistics.

\begin{tabular}{|c|c|c|}
\hline & $\begin{array}{c}\text { RpaI } \\
(\mathrm{CoA}+\text { coumaroyl-SAH })\end{array}$ & MesI \\
\hline \multicolumn{3}{|l|}{ Data collection } \\
\hline Space Group & $\mathrm{P} 2{ }_{1} 2_{1} 2_{1}$ & $\mathrm{P}_{4}{ }_{2}{ }_{1} 2$ \\
\hline Cell: a, b, c $(\AA)$ & $42.52,100.5,105.0$ & $65.7,65.7,113.5$ \\
\hline Resolution $(\AA)^{1}$ & $50-1.85(1.88-1.85)$ & $56.8-1.92(1.93-1.92)$ \\
\hline Total reflections & 234,715 & 513,803 \\
\hline Unique reflections & 38,339 & 19,634 \\
\hline $\mathrm{R}_{\text {sym }}(\%)$ & $11.4(76.5)$ & $4.3(117.6)$ \\
\hline $\mathrm{I} / \sigma(\mathrm{I})$ & $12.5(2.0)$ & $37.9(2.0)$ \\
\hline $\mathrm{CC}(1 / 2)$ & $99.9(0.701)$ & $1.00(0.842)$ \\
\hline Completeness (\%) & $97.4(96.1)$ & 99.8 (94.9) \\
\hline Redundancy & $6.2(6.0)$ & $26.2(21.6)$ \\
\hline \multicolumn{3}{|l|}{ Refinement } \\
\hline Resolution $(\AA)$ & $25.0-1.85$ & $30.0-1.93$ \\
\hline No. reflections & 35,779 & 18,348 \\
\hline $\mathrm{R}_{\text {work }} / \mathrm{R}_{\text {free }}^{2}$ & $18.7 / 22.9$ & $23.2 / 24.6$ \\
\hline \multicolumn{3}{|l|}{ Number of atoms } \\
\hline Protein & 3,389 & 1,558 \\
\hline CoA/Aryl-SAH & $96 / 74$ & \\
\hline Water & 501 & 110 \\
\hline \multicolumn{3}{|l|}{ B-factors } \\
\hline Protein & 20.2 & 40.6 \\
\hline CoA/Aryl-SAH & $22.4 / 19.5$ & \\
\hline Water & 31.5 & 56.8 \\
\hline Molprobity clash score & 5.0 & 11.6 \\
\hline \multicolumn{3}{|l|}{ R.m.s deviations } \\
\hline Bond lengths $(\AA)$ & 0.008 & 0.03 \\
\hline Bond angles $\left({ }^{\circ}\right)$ & 1.49 & 2.1 \\
\hline
\end{tabular}

1. Highest resolution shell is shown in parenthesis.

2. $\mathrm{R}$-factor $=\Sigma\left(\left|\mathrm{F}_{\text {obs }}\right|-\mathrm{k}\left|\mathrm{F}_{\text {calc }}\right|\right) / \Sigma\left|\mathrm{F}_{\text {obs }}\right|$ and R-free is the R value for a test set of reflections consisting of a random $5 \%$ of the diffraction data not used in refinement. 
Table S2. The information of LuxI AHL synthases mentioned in this study.

\begin{tabular}{|c|c|c|}
\hline Protein & UniProt ID & Protein Sequence \\
\hline $\mathrm{BjaI}$ & A0A0N0C224 & $\begin{array}{l}\text { MIHAISAVNRHLYEDVLEQHFRLRHDIFVEERHWETLRRPDGREVDSYD } \\
\text { DEDTVYLLALEGRRVVGGHRLYPTTKPSMMSEVFPHLAAVRGCPSDPLI } \\
\text { WEWSRYFVVRDRRDGALNLQLMAAVQEFCLDQGIAQVSAIMETWWL } \\
\text { PRFHEAGFVVTPLGLPALVENAWTMAATVDIRRQTLDVLHDRIGMPSIV } \\
\text { QQDGPRLDAVARANLCGLAAAQRKSA }\end{array}$ \\
\hline RpaI & Q6NCZ6 & $\begin{array}{l}\text { MQVHVIRRENRALYAGLLEKYFRIRHQIYVVERGWKELDRPDGREIDQ } \\
\text { FDTEDAVYLLGVDNDDIVAGMRMVPTTSPTLLSDVFPQLALAGPVRRP } \\
\text { DAYELSRIFVVPRKRGEHGGPRAEAVIQAAAMEYGLSIGLSAFTIVLET } \\
\text { WWLPRLVDQGWKAKPLGLPQDINGFSTTAVIVDVDDDAWVGICNRRS } \\
\text { VPGPTLEWRGLEAIRRHSLPEFQVIS }\end{array}$ \\
\hline RpaI & A4YLT1 & $\begin{array}{l}\text { MPEIHVVRKDNRALYEKYFDPYYRLRHEIYVKQRKWMDLDRPDGREID } \\
\text { QFDTEDAVYLFCIDNGQLIGSMRAVPTVLPTLMSDIFPYLNLRGPVQRP } \\
\text { DVYELSRIFVIPERRGEHAGPRIDMLLLTAIMEYGISIGLTGFSIVLESWW } \\
\text { LPRFEKCGWKARPLGVPHIMDGMSVLAVLVDCDETTWKSLCTQIGLTR } \\
\text { PTLTWQGLEEVSRQALPDIFLHLPPAVQPAQ }\end{array}$ \\
\hline BolI & M4ZFS5 & $\begin{array}{l}\text { MPEIHVVRKDNRALYEKYFDPYYRLRHEIYVKQRKWMALDRPDGREID } \\
\text { QFDTEESVYLFCIDKGQLIGSMRAVPTLMPTLMSDIFPYLNLRGQIQRPD } \\
\text { VYELSRIFVIPERRGEHAGPRVDLLLLTAIMEYGISIGLTGFSIVLETWWL } \\
\text { PRFEKCGWKARPLGVPHMMDGMSVMAVLVDCDETTWKSLCTQIGLTR } \\
\text { PTLTWQGLEDVSRQALPDIFLQLPPAFQPAQ }\end{array}$ \\
\hline MesI & A0A090F1W4 & $\begin{array}{l}\text { MVRIHLVTWENRKLYRKVLERYFRIRYDIYVKQRRWRAVARPINIEIDA } \\
\text { FDNEHALYVLALDANGKIVGGSRLVPTLEPHLMSEVFPILAGGTPPRAA } \\
\text { EIFEWTRFFVMPSFRTKGASSPVAGFVLCGLLETAQSLGIRQISVVCETF } \\
\text { WPKRLRALGWTLFELGNALEHPDGDIIALLIDVTPEAIEQTRRAYGISGA } \\
\text { ILADGI }\end{array}$ \\
\hline MplI & A0A090GMF8 & $\begin{array}{l}\text { MITAHVVNAQNRHLYENEFDEFLRRRHDFFVHQKRWRPPSPDGRELDQ } \\
\text { FDTDAATYLLGMEEGRVVTSARLIPTSEPHMVSEVFPHMCEKSGVPRRP } \\
\text { DWAEWTRTFVVPEKRSTGLRGTLTQLCAVMEYALDEGLSAVGGVQE } \\
\text { TYFMPHHGALKWHAKPLGMAREENGEWYIVAYIEVNEAALASVRKIL } \\
\text { GLDRSLLVRRGIQLPFVKVNRHLAEVAR }\end{array}$ \\
\hline LasI & A0A1C7BUJ1 & $\begin{array}{l}\text { MIVQIGRREEFDKKLLGEMHKLRAQVFKERKGWDVSVIDEMEIDGYDA } \\
\text { LSPYYMLIQEDTPEAQVFGCWRILDTTGPYMLKNTFPELLHGKEAPCSP } \\
\text { HIWELSRFAINSGQKGSLGFSDCTLEAMRALARYSLQNDIQTLVTVTTV } \\
\text { GVEKMMIRAGLDVSRFGPHLKIGIERAVALRIELNAKTQIALYGGVLVE } \\
\text { QRLAVS }\end{array}$ \\
\hline EsaI & P54656 & $\begin{array}{l}\text { MLELFDVSYEELQTTRSEELYKLRKKTFSDRLGWEVICSQGMESDEFDG } \\
\text { PGTRYILGICEGQLVCSVRFTSLDRPNMITHTFQHCFSDVTLPAYGTESS } \\
\text { RFFVDKARARALLGEHYPISQVLFLAMVNWAQNNAYGNIYTIVSRAML } \\
\text { KILTRSGWQIKVIKEAFLTEKERIYLLTLPAGQDDKQQLGGDVVSRTGC } \\
\text { PPVAVTTWPLTLPV }\end{array}$ \\
\hline
\end{tabular}


Table S3 Primers used for generating variants of RpaI, MesI, MpII, and BjaI. All sequences provided 5' to 3', "F" indicates a forward primer, " $\mathrm{R}$ " indicates reverse primer.

\begin{tabular}{ll}
\hline Primer name & Primer sequence \\
\hline Rpal-LIC-F & TACTTCCAATCCAATGCAATGCAGGTTCATGTCATCCG \\
\hline Rpal-LIC-R & TTATCCACTTCCAATGTTATTATGAAATCACCTGGAATTCCGGAAGCG \\
\hline Rpal-Q124V-F & GAAGCCGTGATCGTGGCGGCCGCGATGG \\
Rpal-Q124V-R & CCATCGCGGCCGCCACGATCACGGCTTC \\
Mesl-LIC-F & TACTTCCAATCCAATGCAATGGTGAGAATCCATCTTGTTACC \\
Mesl-LIC-R & TTATCCACTTCCAATGTTATTAGATTCCGTCGGCCAGAATTGC \\
MesI-L153A-F & CTTTTTGGCCTAAACGCGCACGGGCTTTGGGTTGG \\
MesI-L153A-R & CCAACCCAAAGCCCGTGCGCGTTTAGGCCAAAAAG \\
MesI-L153F-F & CTTTTTGGCCTAAACGCTTTCGGGCTTTGGGTTGG \\
MesI-L153F-R & CCAACCCAAAGCCCGAAAGCGTTTAGGCCAAAAAG \\
MplI-LIC-F & TACTTCCAATCCAATGCAATGATAACTGCGCATGTCGTAAACG \\
MplI-LIC-R & TTATCCACTTCCAATGTTATTAACGAGCGACCTCGGCCAGG \\
MplI-T105Y-F & GCGGAGTGGACTAGATACTTCGTGGTACCCGAG \\
MplI-T105Y-R & CTCGGGTACCACGAAGTATCTAGTCCACTCCGC \\
Bjal-LIC-F & TACTTCCAATCCAATGCAATGATTCACGCAATTTCCGCGG \\
Bjal-LIC-R & TTATCCACTTCCAATGTTATTAGGCGCTCTTTCGTTGCGCG \\
Bjal-F147Y-F & GTGGTTGCCGCGCTatCACGAGGCCGGCT \\
Bjal-F147Y-R & AGCCGGCCTCGTGatAGCGCGGCAACCAC \\
\hline
\end{tabular}




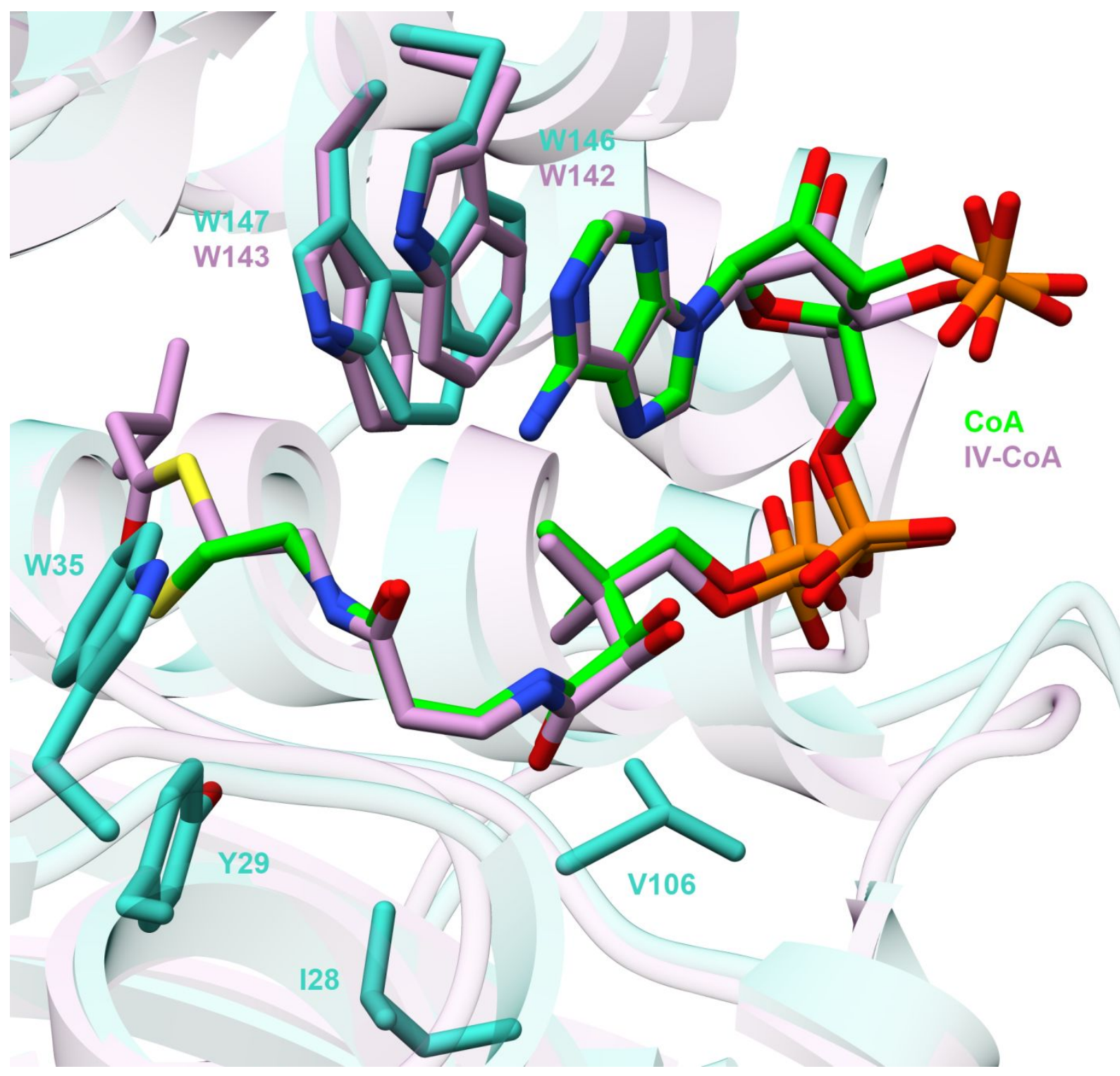

Figure S1. Closeup view of the overlay of CoA binding site of RpaI (in complex with CoA) and BjaI (in complex with IV-CoA). Ribbon diagram of RpaI structure is colored in turquoise and that of $\mathrm{BjaI}$ is colored in plum. The side chains of hydrophobic residues aligning CoA, including Ile 28, Tyr29, Trp35, Val106, Trp 146, and Trp147 are shown in sticks and marked in the corresponding colors. 


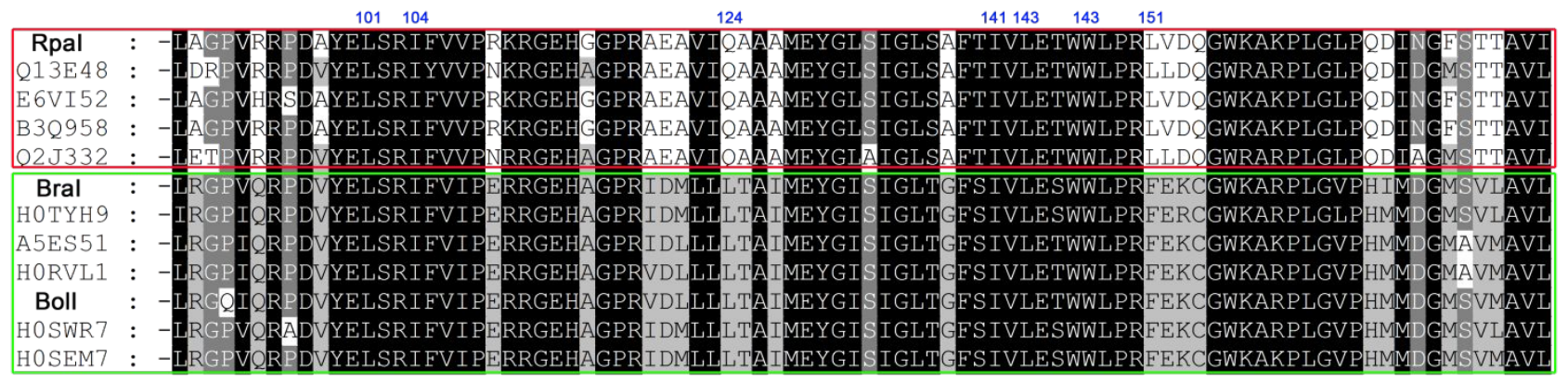

Figure S2. Sequence alignment analysis of cluster 1b. Different sequences with notable variance of key residues involved in acyl group binding are labeled by rectangles with different colors. The residue numbers are marked representing RpaI residues. 


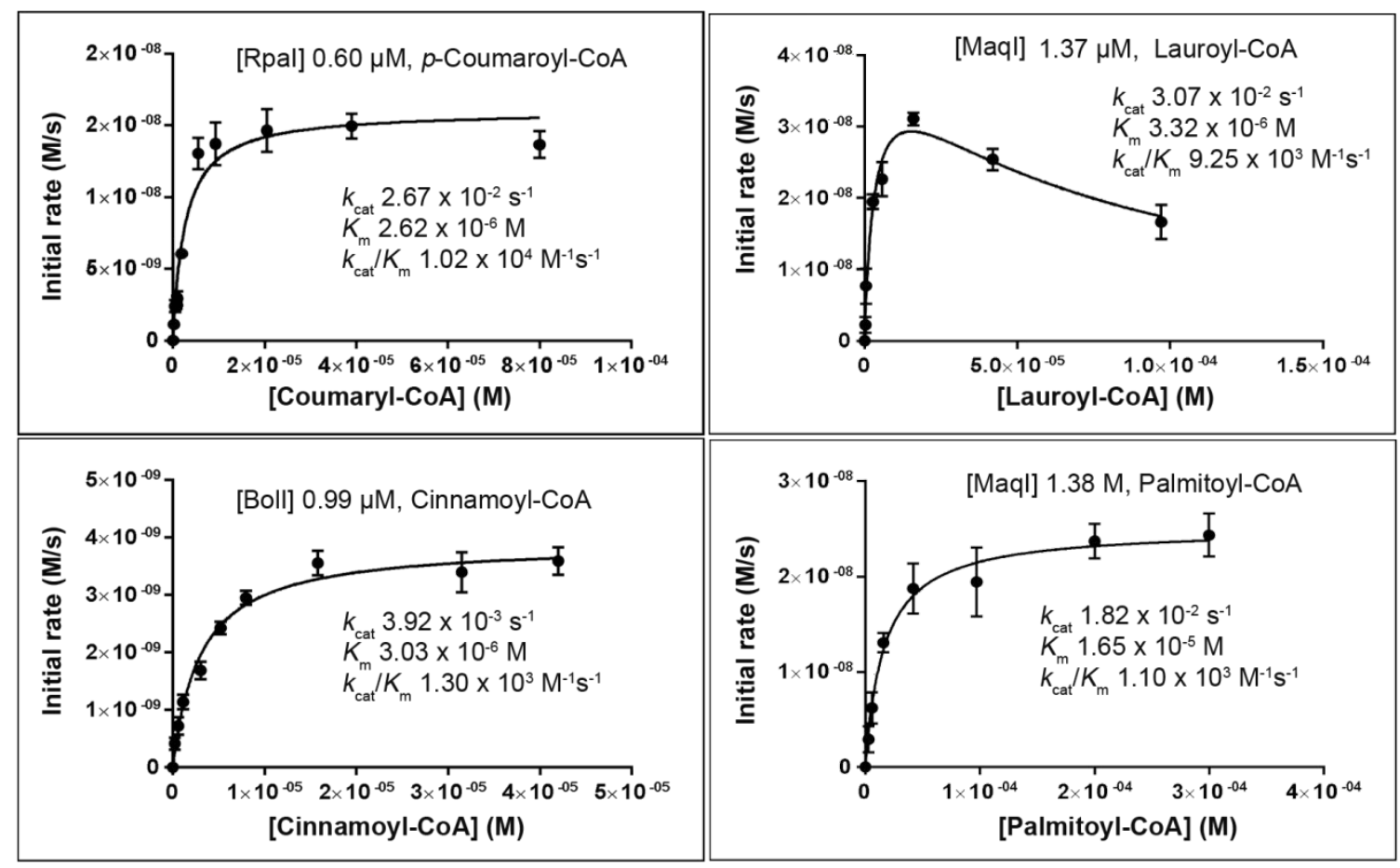

Figure S3. The kinetics curves and parameters of RpaI, BolI, and MaqI. The enzyme concentration and the identity of the acyl-CoA used in the assay are marked on the top of the corresponding curve. 

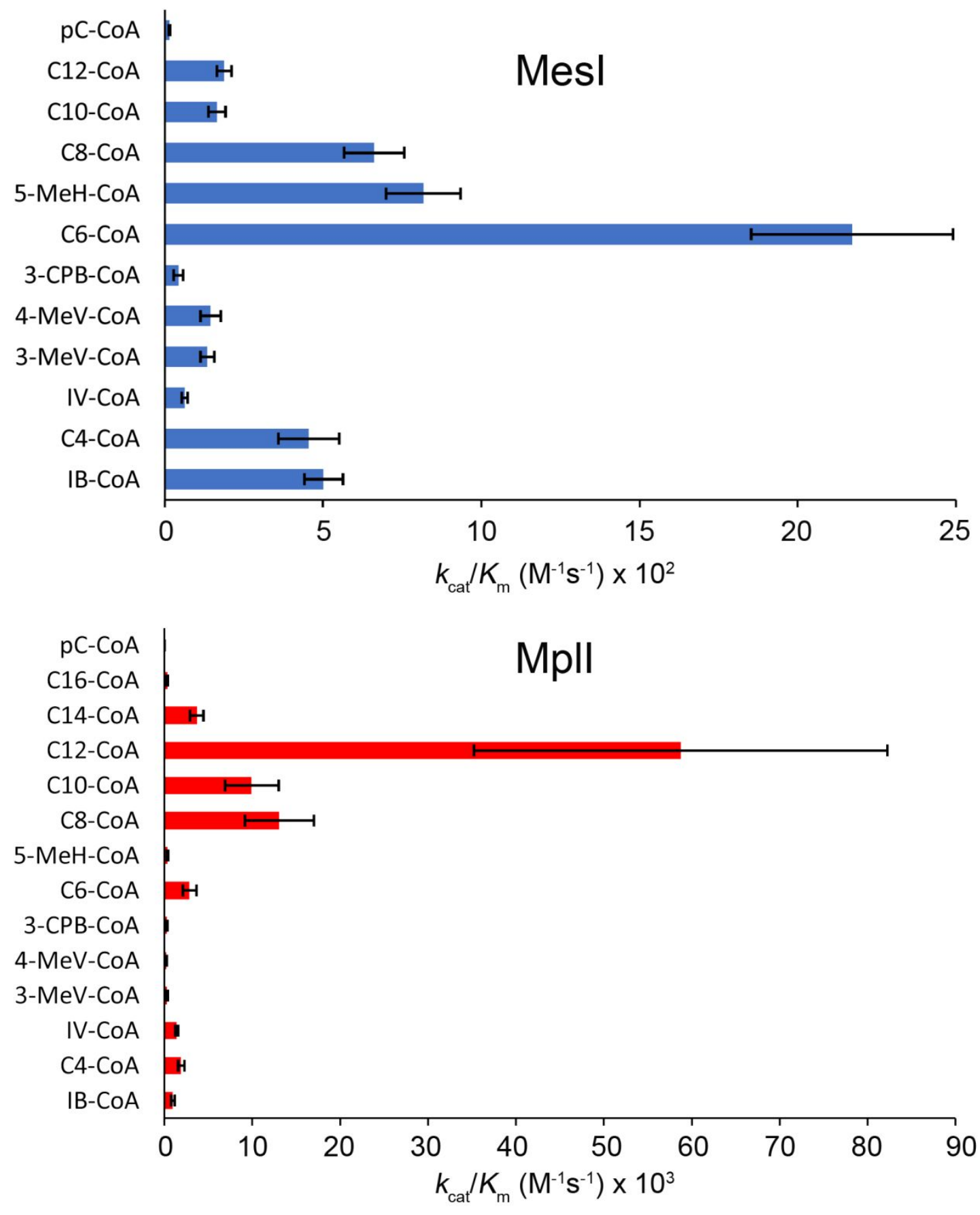

Figure S4. The bar diagrams of catalytic efficiency $\left(k_{\mathrm{cat}} / K_{\mathrm{m}}\left(\mathrm{M}^{-1} \mathrm{~s}^{-1}\right) \times 10^{3}\right)$ of MesI and MplI. The data was acquired from Table S1. 


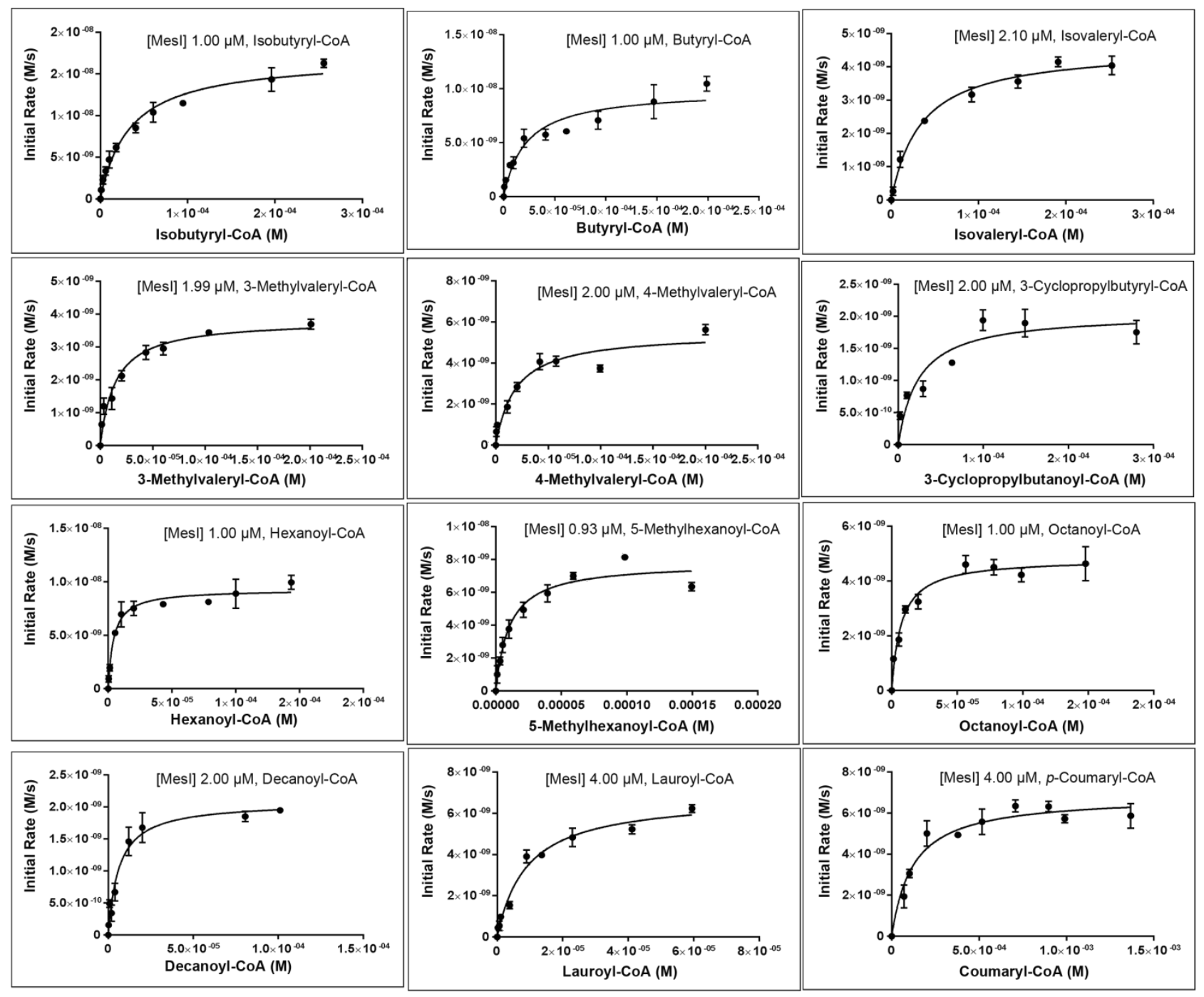

Figure S5. The kinetics curves of MesI. The MesI concentration and the identity of the acyl-CoA used in the assay are marked on the top of the corresponding curve. 


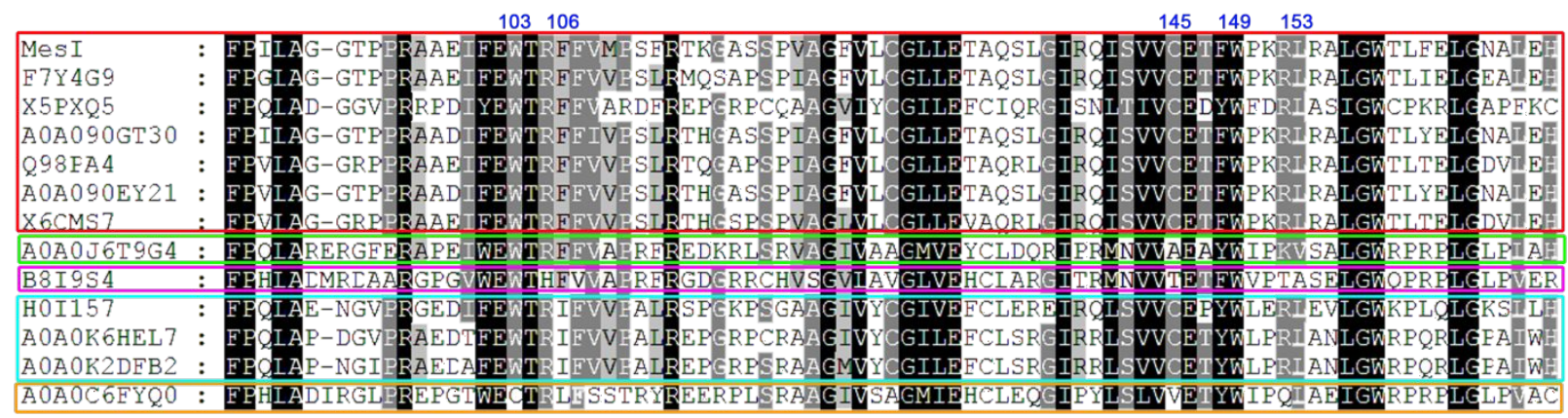

Figure S6. Sequence alignment analysis of cluster 1c. Different sequences with notable variance of key residues involved in acyl group binding are labeled by rectangles with different colors. The residue numbers are marked representing MesI residues. 

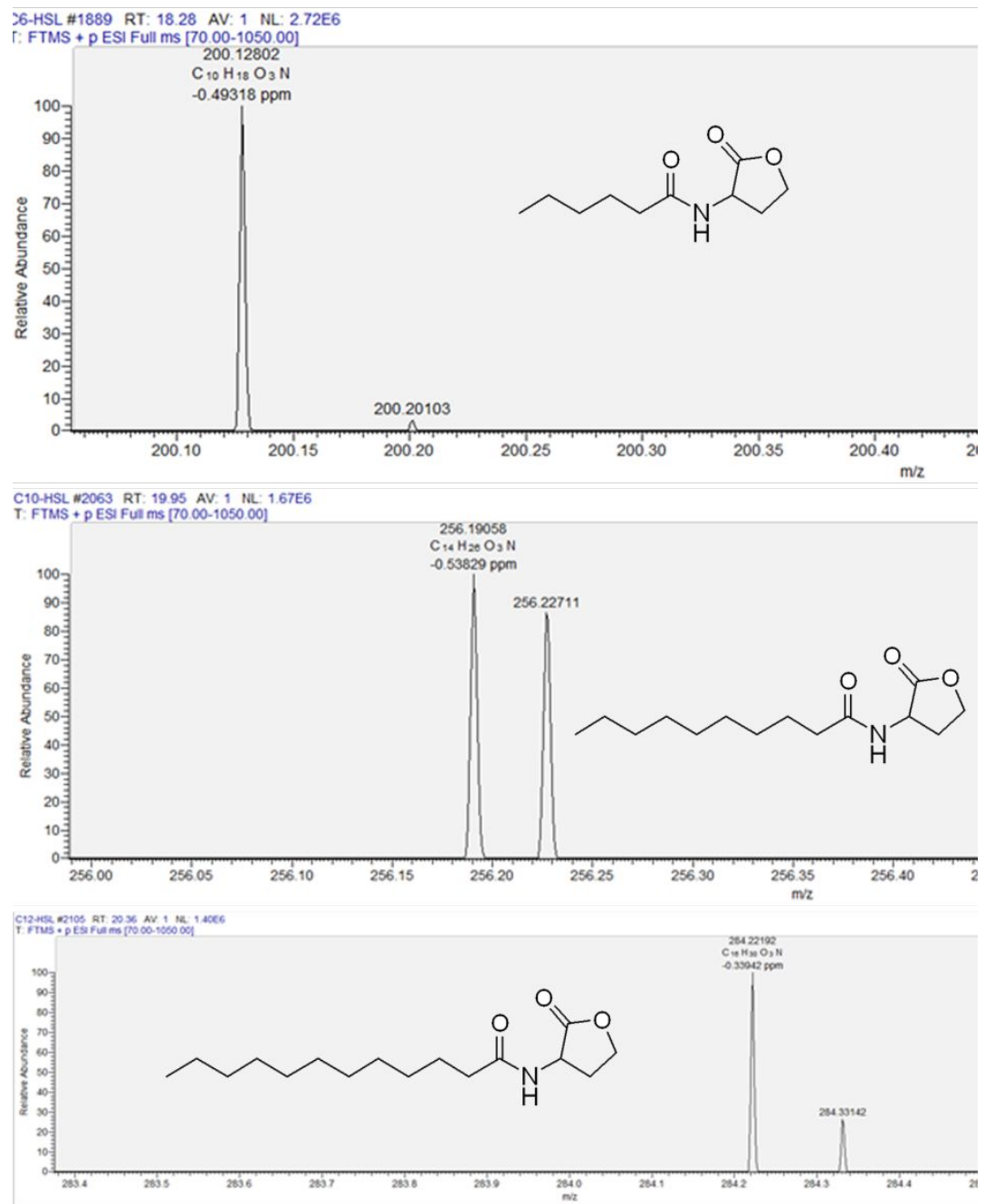

Figure S7. High Resolution LC-MS analysis of MaqI reaction using various acyl-CoAs. MaqI assay was carried out with $\mathrm{C} 6 / \mathrm{C} 10 / \mathrm{C} 12-\mathrm{CoAs}$ using previous conditions, ${ }^{1}$ and reactions were incubated at $37{ }^{\circ} \mathrm{C}$ for $2 \mathrm{hrs}$ before subjecting to high resolution LC-MS analysis. Peaks indicated with corresponding theoretical molecular formulas represent the high-resolution mass of corresponding acyl-HSL. 


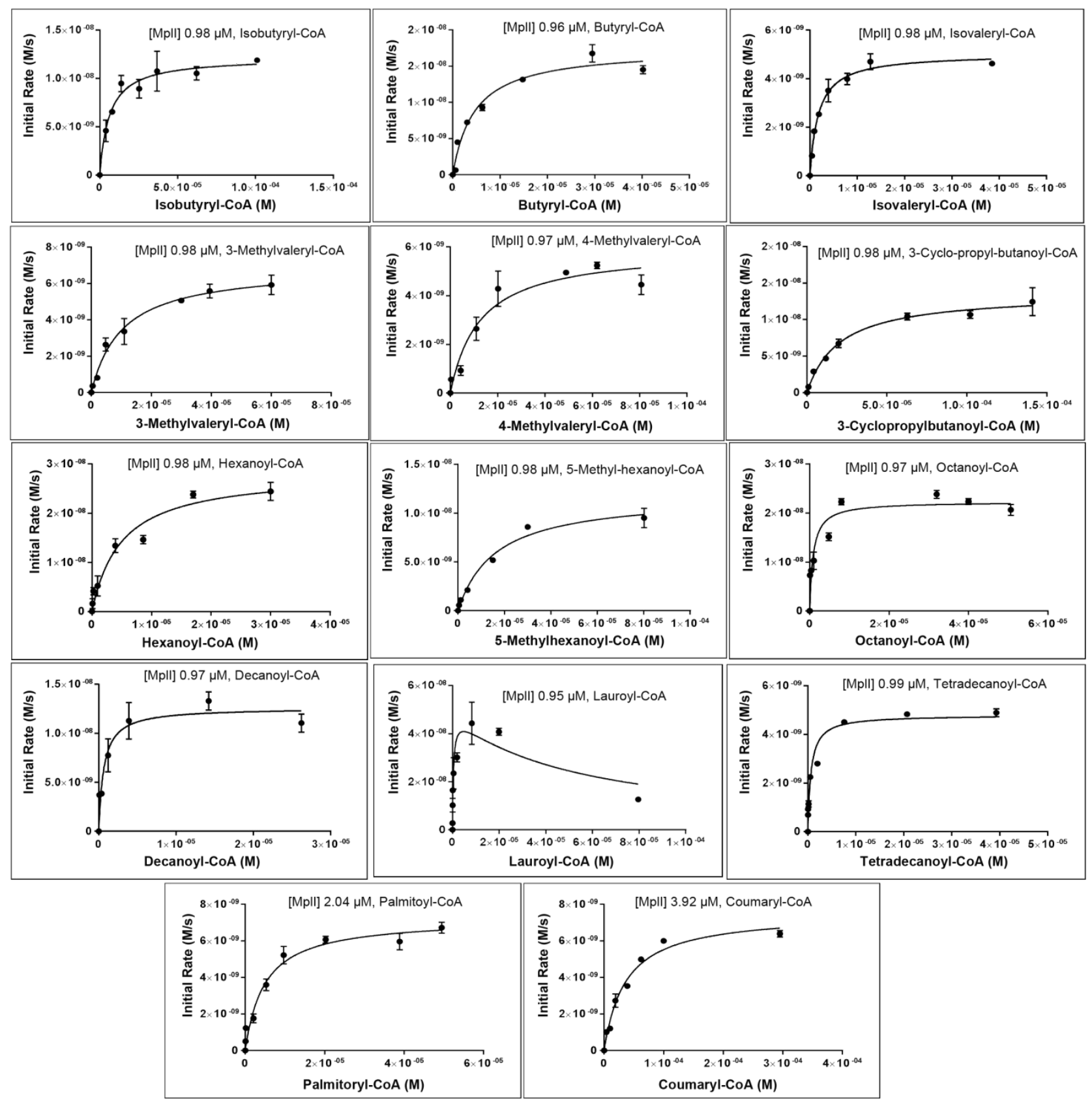

Figure S8. The kinetics curves of MplI. The MplI concentration and the identity of the acyl-CoA used in the assay are marked on the top of the corresponding curve. 
102105

$143 \quad 147 \quad 151$

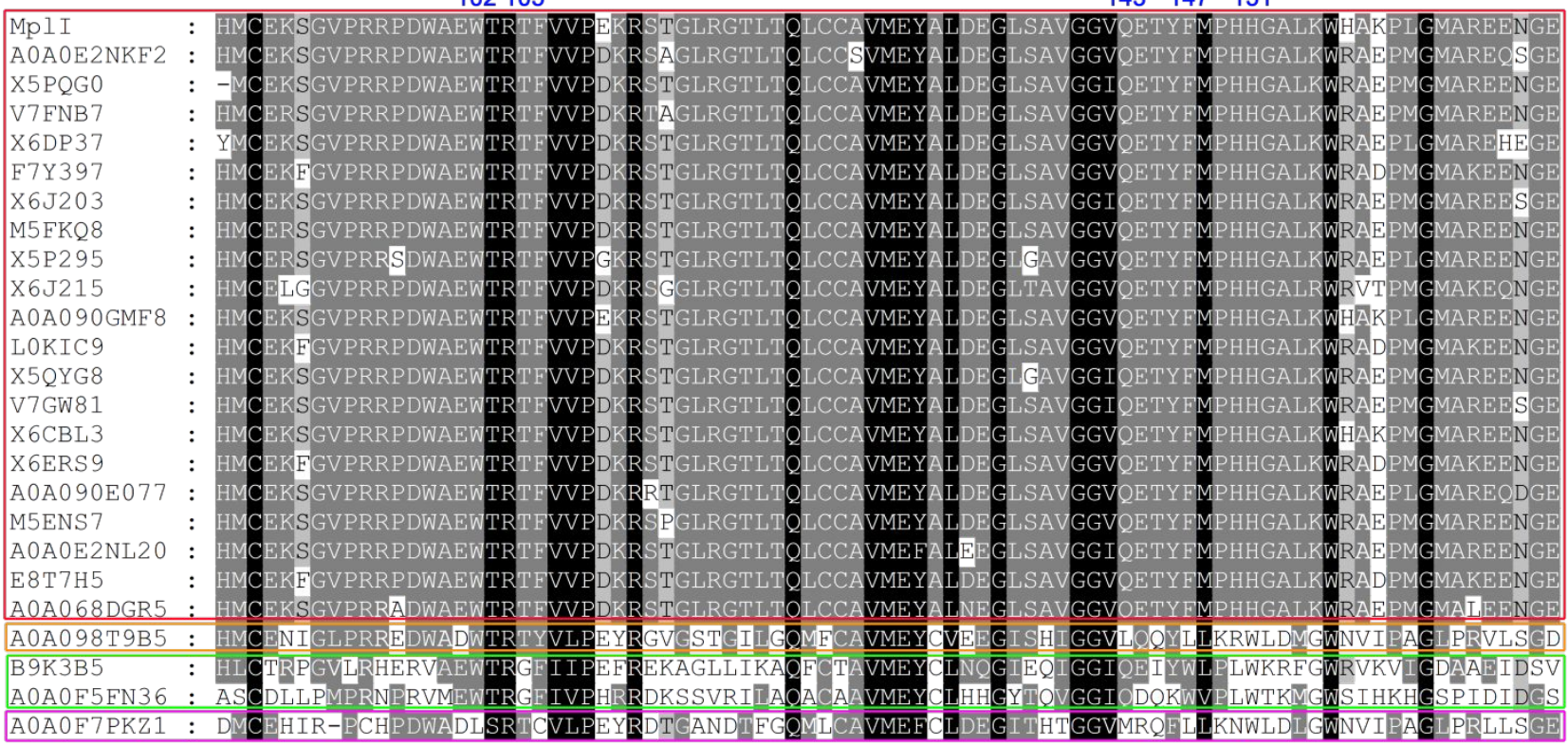

Figure S9. Sequence alignment analysis of cluster 2. Different sequences with notable variance of key residues involved in acyl group binding are labeled by rectangles with different colors. The residue numbers are marked representing MplI residues. 

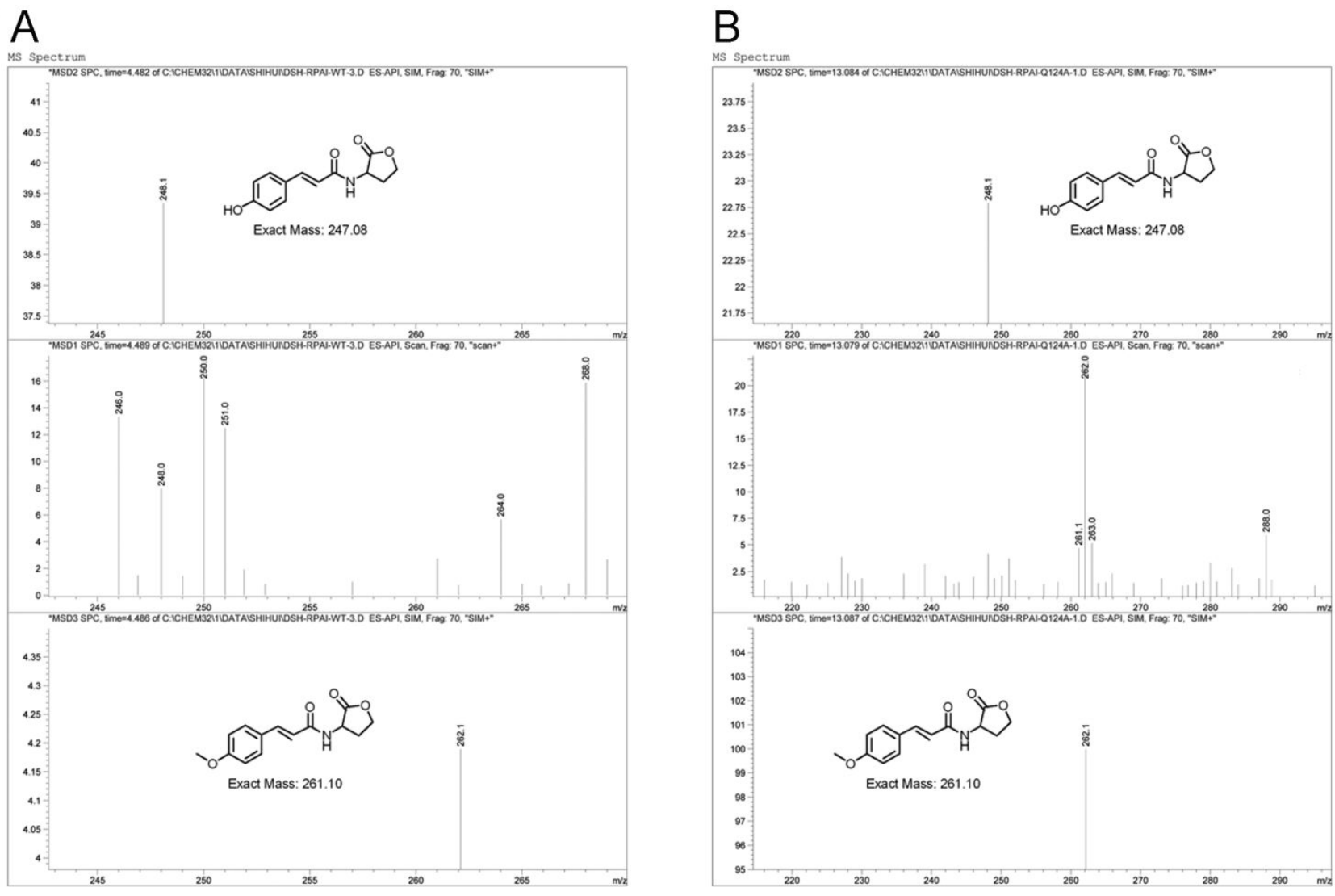

Figure S10. ESI mass spectra of RpaI (A) and its Q124A variant (B) reactions using mixture of $p \mathrm{C}$ - and Me-pC-CoAs as substrates. The corresponding AHL structures were marked on top of the ESI spectra using SIM mode, and their intensities were used for quantification. The scan mode spectra were attached to show the presence of both products. 

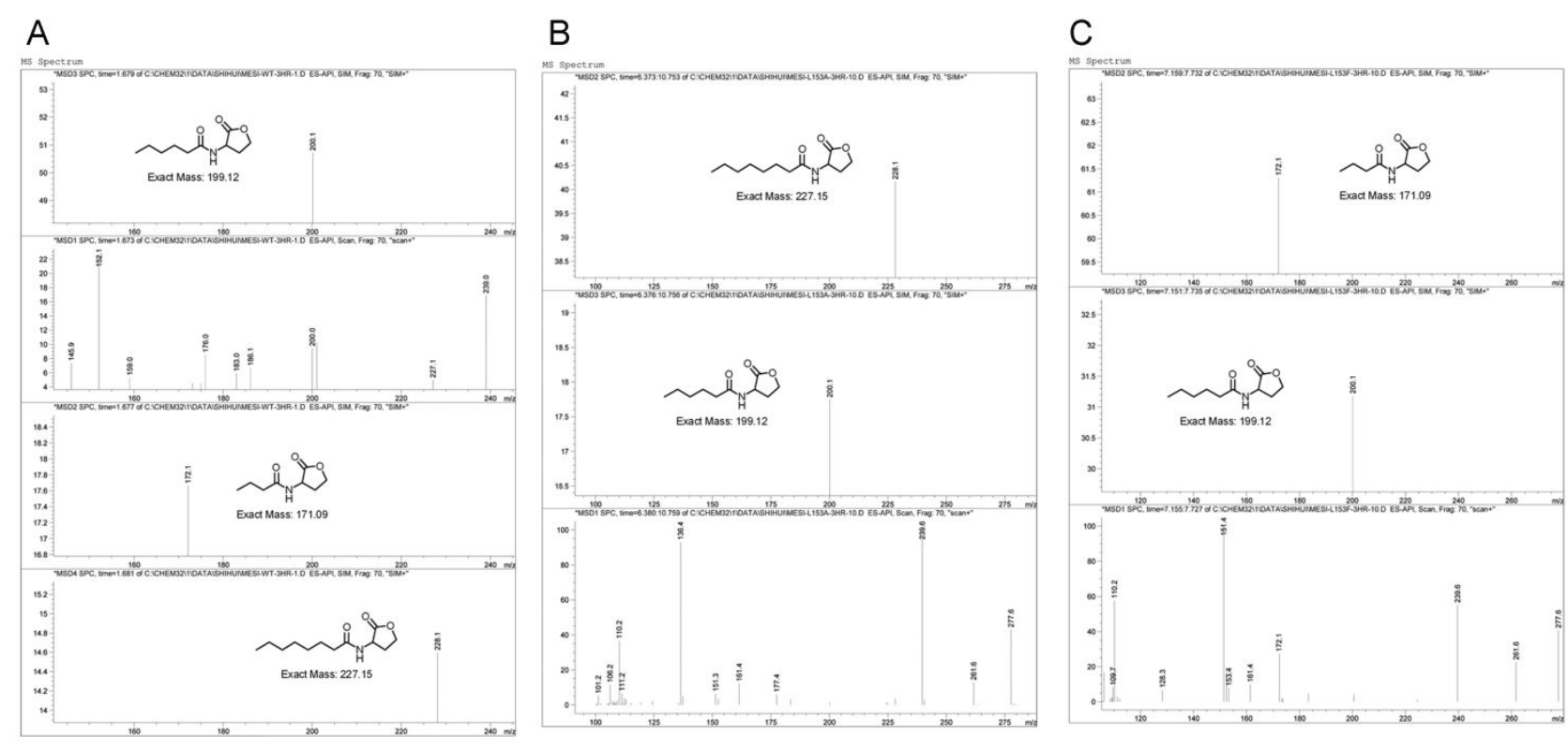

Figure S11. ESI mass spectra of MesI (A) and its L153A (B) and L153F (C) variants reactions using mixture of C6-, C4-, and/or C8-CoAs as substrates. The corresponding AHL structures were marked on top of the ESI spectra using SIM mode, and their intensities were used for quantification. The scan mode spectra were attached to show the presence of both products. 

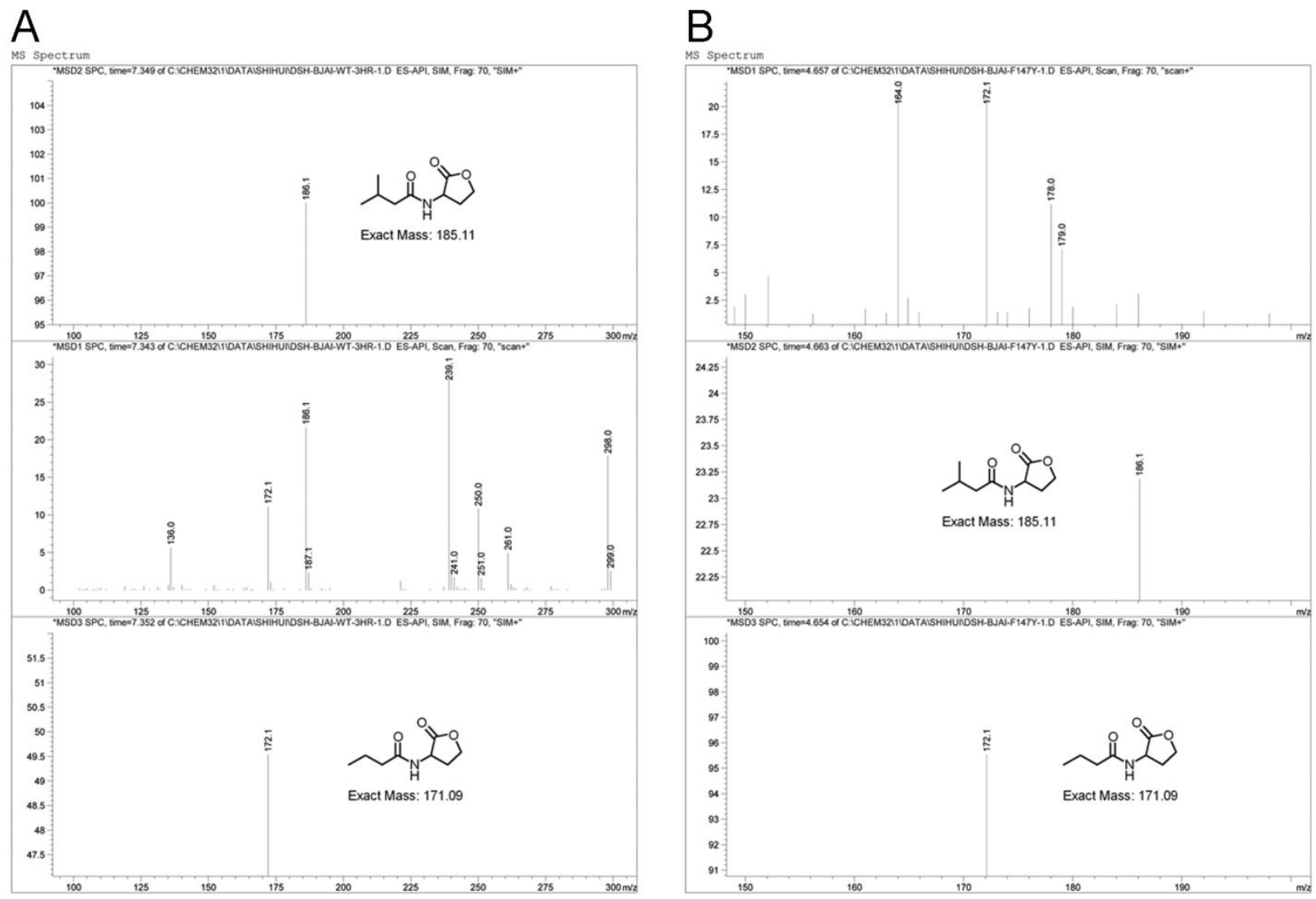

Figure S12. ESI mass spectra of BjaI (A) and its F147Y variant (B) reactions using mixture of IV- and C4-CoAs as substrates. The corresponding AHL structures were marked on top of the ESI spectra using SIM mode, and their intensities were used for quantification. The scan mode spectra were attached to show the presence of both products. 

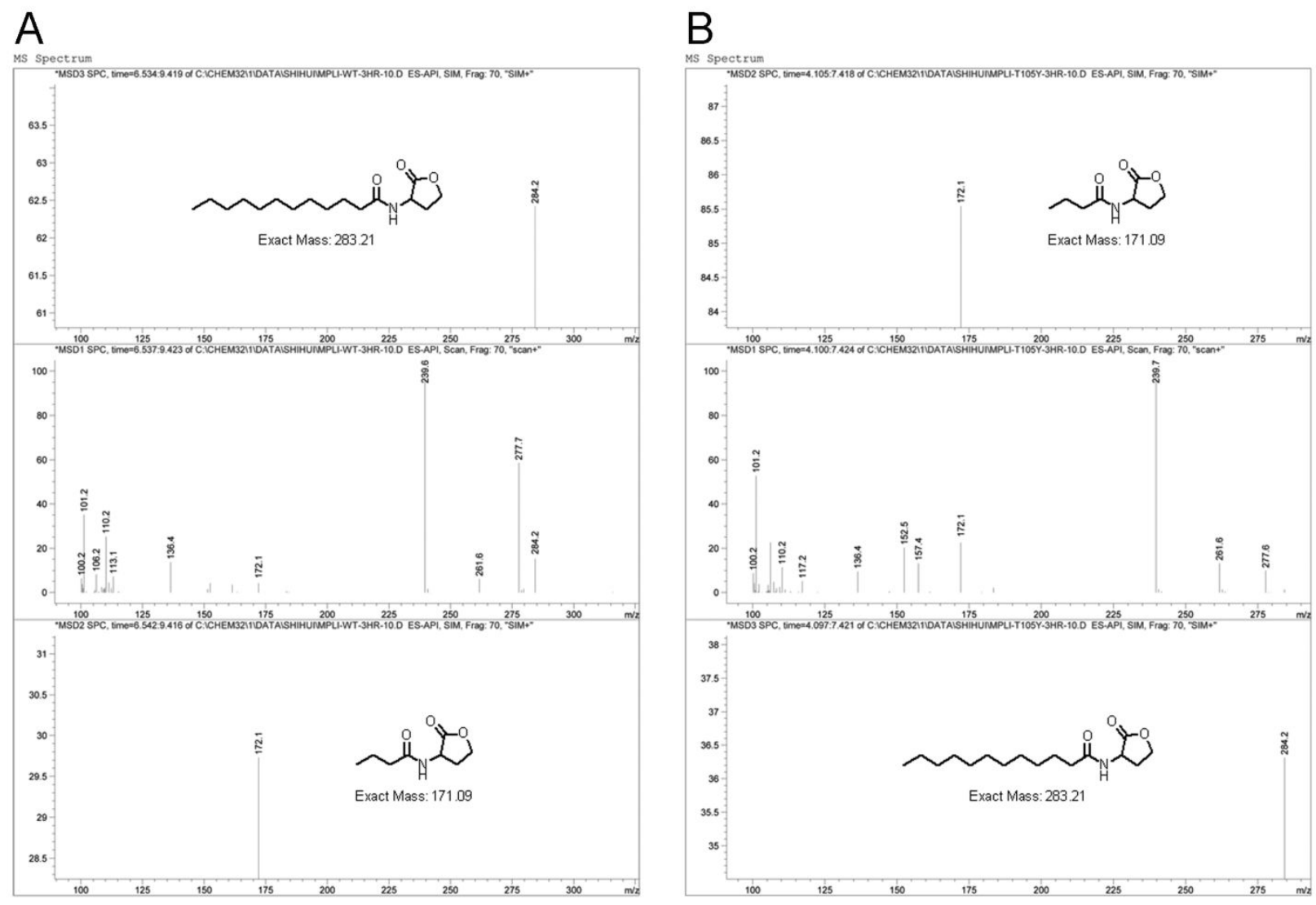

Figure S13. ESI mass spectra of MpII (A) and its T105Y variant (B) reactions using mixture of C12- and C4-CoAs as substrates. The corresponding AHL structures were marked on top of the ESI spectra using SIM mode, and their intensities were used for quantification. The scan mode spectra were attached to show the presence of both products. 


\section{REFERENCES}

(1) Dong, S. H.; Frane, N. D.; Christensen, Q. H.; Greenberg, E. P.; Nagarajan, R.; Nair, S. K. Molecular basis for the substrate specificity of quorum signal synthases. Proc Natl Acad Sci U S A 2017, 114, 90929097.

(2) Otwinowski, Z.; Borek, D.; Majewski, W.; Minor, W. Multiparametric scaling of diffraction intensities. Acta Crystallogr A 2003, 59, 228-234.

(3) Kabsch, W. Processing of X-ray snapshots from crystals in random orientations. Acta Crystallogr D Biol Crystallogr 2014, 70, 2204-2216.

(4) McCoy, A. J. Solving structures of protein complexes by molecular replacement with Phaser. Acta Crystallogr D Biol Crystallogr 2007, 63, 32-41.

(5) Afonine, P. V.; Grosse-Kunstleve, R. W.; Echols, N.; Headd, J. J.; Moriarty, N. W.; Mustyakimov, M.; Terwilliger, T. C.; Urzhumtsev, A.; Zwart, P. H.; Adams, P. D. Towards automated crystallographic structure refinement with phenix.refine. Acta Crystallogr D Biol Crystallogr 2012, 68, 352-367.

(6) Emsley, P.; Cowtan, K. Coot: model-building tools for molecular graphics. Acta Crystallogr D Biol Crystallogr 2004, 60, 2126-2132.

(7) Brunger, A. T. Free R value: cross-validation in crystallography. Methods Enzymol 1997, 277, 366-396.

(8) Laskowski, R. A.; Rullmannn, J. A.; MacArthur, M. W.; Kaptein, R.; Thornton, J. M. AQUA and PROCHECK-NMR: programs for checking the quality of protein structures solved by NMR. J Biomol NMR 1996, $8,477-486$.

(9) Waterhouse, A.; Bertoni, M.; Bienert, S.; Studer, G.; Tauriello, G.; Gumienny, R.; Heer, F. T.; de Beer, T. A. P.; Rempfer, C.; Bordoli, L.; Lepore, R.; Schwede, T. SWISS-MODEL: homology modelling of protein structures and complexes. Nucleic Acids Res 2018, 46, W296-W303.

(10) Benkert, P.; Biasini, M.; Schwede, T. Toward the estimation of the absolute quality of individual protein structure models. Bioinformatics 2011, 27, 343-350.

(11) Christensen, Q. H.; Brecht, R. M.; Dudekula, D.; Greenberg, E. P.; Nagarajan, R. Evolution of acylsubstrate recognition by a family of acyl-homoserine lactone synthases. PLoS One 2014, 9, e112464.

(12) Shin, D.; Frane, N. D.; Brecht, R. M.; Keeler, J.; Nagarajan, R. A Comparative Analysis of AcylHomoserine Lactone Synthase Assays. ChemBioChem 2015, 16, 2651-2659. 